\title{
Carbothermal Processing of Lunar Regolith Using Methane
}

\author{
R. Balasubramaniam ${ }^{1}$, U. Hegde ${ }^{1}$ and S. Gokoglu ${ }^{2}$ \\ ${ }^{1}$ National Center for Space Exploration Research (NCSER), NASA Glenn Research Center, Cleveland, Ohio 44135 \\ ${ }^{2}$ NASA Glenn Research Center, Cleveland, Ohio 44135 \\ Tel:(216)433-2878, email: bala@grc.nasa.gov
}

\begin{abstract}
The processing of lunar regolith for the production of oxygen is a key component of the In-Situ Resource Utilization plans currently being developed by NASA. Among various candidate processes, the modeling of oxygen production by hydrogen reduction, molten salt electrolysis, and carbothermal processing are presently being pursued. In the carbothermal process, a portion of the surface of the regolith in a container is heated by exposure to a heat source such as a laser beam or a concentrated solar heat flux, so that a small zone of molten regolith is established. The molten zone is surrounded by solid regolith particles that are poor conductors of heat. A continuous flow of methane is maintained over the molten regolith zone. Our model is based on a mechanism where methane pyrolyzes when it comes in contact with the surface of the hot molten regolith to form solid carbon and hydrogen gas. Carbon is deposited on the surface of the melt, and hydrogen is released into the gas stream above the melt surface. We assume that the deposited carbon mixes in the molten regolith and reacts with metal oxides in a reduction reaction by which gaseous carbon monoxide is liberated. Carbon monoxide bubbles through the melt and is released into the gas stream. Oxygen is produced subsequently by (catalytically) processing the carbon monoxide downstream. In this paper, we discuss the development of a chemical conversion model of the carbothermal process to predict the rate of production of carbon monoxide.
\end{abstract}

Keywords: Carbothermal processing, lunar regolith, pyrolysis, kinetics

PACS: 47.70.Fw, 82.40.-g

\section{INTRODUCTION}

NASA's plans to return to the Moon involves the production of oxygen via In-Situ Resource Utilization, for life support as well as use as a propellant. Oxygen is available in the lunar regolith as oxides of various materials. The extraction of gaseous oxygen from the oxides by reduction using hydrogen (Taylor and Carrier, 1992), molten salt electrolysis (Tripuraneni-Kilby et al., 2006) and carbothermal processing of regolith (ORBITEC, 2006) are currently being pursued by NASA (Sanders, Larson and Sacksteder, 2007).

Carbothermal processing refers to the use of carbon in some form, together with high processing temperature, to reduce the oxides. Examples of carbothermal processes on Earth are the production of silicon from silica using wood, charcoal and coal (a standard process), and the production of titanium-rich materials by refining low-grade ilmenites using graphite (Wang and Yuan, 2006).

The carbothermal processing of lunar regolith using methane as the source for carbon was first performed by Rosenberg and co-workers at Aerojet in the 1960's (Rosenberg et al., 1991). It has been revisited by researchers at Orbital Technologies Corporation (ORBITEC) since the early 1990's. An excellent reference and resource for the application of the carbothermal process for the conversion of lunar regolith to ultimately produce oxygen is the Phase 1 final report by ORBITEC (ORBITEC, 2006). ORBITEC's objective was to provide proof of concept hardware for the transport of regolith into and out of the reactor, to determine reaction time for oxygen extraction and carbon recovery rates, and to perform low-pressure carbothermal reduction experiments.

Our objective is to model the production of carbon monoxide in the carbothermal reactor, and predict the rate at which $\mathrm{CO}$ is liberated as a function of the various physicochemical parameters of the system. We consider the conversion of 


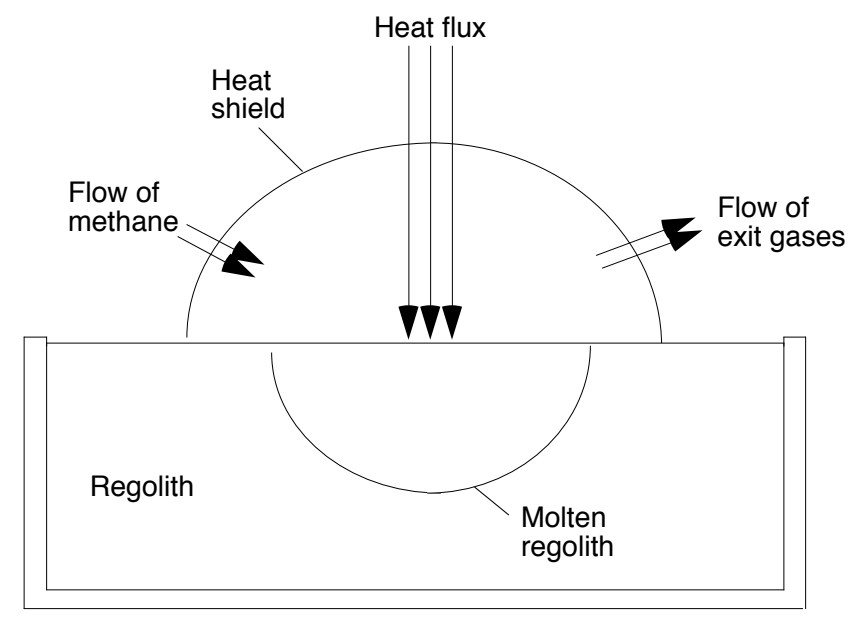

FIGURE1: Sketch of the Carbothermal Reduction of Lunar Regolith.

lunar regolith in a carbothermal reactor through which methane flows continuously. Figure 1 shows a schematic sketch of the carbothermal reactor. Lunar regolith is contained in a suitable container. A portion of the surface of the regolith is heated by exposure to a heat source such as a laser beam or a concentrated solar heat flux, so that a small zone of molten regolith is established within the container. A heat shield is used above the molten zone to minimize radiative heat loss. A continuous flow of methane is maintained over the molten regolith zone. Since the molten regolith is very hot (temperature will exceed $1650^{\circ} \mathrm{C}$ ), methane will pyrolyze when it contacts the molten zone. Carbon is deposited on the surface of the melt, and hydrogen is released into the gas phase above the melt surface. The deposited carbon reacts with metal oxides (chiefly $\mathrm{SiO}_{2}$ ) in the molten regolith in a reduction reaction in which carbon monoxide is liberated. $\mathrm{CO}$ bubbles through the melt and is released in the gas phase above the melt. It is processed in a catalytic reactor downstream at a lower temperature, where it combines with hydrogen to form water and methane. Methane is recycled, and the water is electrolyzed further downstream to yield oxygen and hydrogen. Oxygen is liquified and stored for use as a propellant and for life support, and the hydrogen is recycled to the catalytic reactor.

\section{MODEL FORMULATION AND ASSUMPTIONS}

We assume that carbon deposition occurs on the hot molten surface via a surface reaction. This surface reaction is regarded as first order with respect to a gaseous carbon-containing species and produces solid carbon and gaseous hydrogen. This carbon-containing species need not necessarily be methane; it can be some other carboneous species which may be produced by the gas-phase pyrolysis of methane. In reality, depending on the operating pressure, flow conditions and temperature profiles above the hot molten surface, there would be a distribution of carbon-containing species (e.g., methane, ethylene, acetylene, methyl radical, etc.,) and each of them would react on the surface to deposit carbon at their own rates. Our model, however, "lumps" all of these possibilities into a "single" species and describes the deposition process by a first-order surface reaction of this "predominant" species. For sake of simplicity, we call this "lumped" species methane throughout the paper. Thus the flux of carbon deposited on the surface of the melt is assumed to be proportional to the concentration of methane in the gas phase above the melt. The proportionality constant needs to be determined by comparison of the model predictions with experimental data. The concentration of methane above the melt is assumed constant by its continuous flow in the gas phase.

The deposited carbon is assumed to undergo a single step, first order chemical reaction with the metal oxide present in the molten regolith. This reaction rate constant also needs to be determined by comparison of the model predictions with experimental data. Our simplified model does not account for spatial gradients of the concentration of carbon or the metal oxide within the melt; the zone is regarded as 'well-mixed.' This would be the case when the zone is 
sufficiently small that diffusion of carbon within the melt is rapid, or more likely, when there is flow within the melt caused by the evolution of bubbles, natural convection, and thermocapillary forces.

We assume that the molten zone is established and maintained by heating the regolith by suitable means. The heat transfer aspects of the problem are not analyzed here. The processes of chemical conversion and heat transfer within the molten regolith are assumed to be uncoupled.

Let $\alpha^{*}$ and $\beta^{*}$ denote the molar concentration (moles $/ \mathrm{m}^{3}$ ) of carbon and metal oxide, respectively, within the melt. At time $t^{*}=0$, the initial concentrations are $\alpha^{*}=0$ and $\beta^{*}=\beta_{0}$. Let $V$ and $A$ denote the volume and the surface area of the molten zone of regolith. They are assumed to be constant and independent of time during the chemical conversion process. The chemical reaction between carbon and the metal oxide in the regolith is

$$
a M O_{x}+C \Leftrightarrow C O+a M
$$

The stoichiometric coefficient of the oxide term in the above reaction is $a=1 / x$. We assume that the chemical reaction is first order with respect to carbon and the metal oxide, with a rate constant $k\left(\mathrm{~m}^{3} /(\right.$ moles s) $)$. The flux of carbon due to pyrolysis of methane is denoted by $r_{p}$, and is assumed to be linearly proportional to the molar concentration of methane $C_{m}$ in the gas phase adjacent to the melt surface, with the constant of proportionality denoted by $\gamma(\mathrm{m} / \mathrm{s})$. The equations for the evolution of $\alpha^{*}$ and $\beta^{*}$ in the melt can be obtained from mass conservation to be:

$$
\begin{gathered}
\frac{d \alpha^{*}}{d t^{*}}=\frac{A}{V} r_{p}-k \alpha^{*} \beta^{*}=\frac{A \gamma C_{m}}{V}-k \alpha^{*} \beta^{*}, \\
\frac{d \beta^{*}}{d t^{*}}=-a k \alpha^{*} \beta^{*} .
\end{gathered}
$$

Dimensionless variables are defined as follows.

$$
\alpha=\frac{\alpha^{*}}{\beta_{0} / a} \quad \beta=\frac{\beta^{*}}{\beta_{0}} \quad t=\frac{t^{*}}{1 /\left(k \beta_{0}\right)} .
$$

A dimensionless parameter that appears in the equations when they are cast in terms of these dimensionless quantities is $\lambda=\frac{a A \gamma C_{m}}{k V \beta_{0}^{2}}$. The instantaneous rate of production of carbon monoxide is

$$
\frac{d X_{C O}}{d t^{*}}=-\frac{V}{a} \frac{d \beta^{*}}{d t^{*}}=\frac{V k \beta_{0}^{2}}{a} \alpha \beta
$$

\section{RESULTS}

Plots of the scaled concentrations of carbon $(\alpha)$ and metal oxide $(\beta)$, and the scaled rate of production of carbon monoxide (which is proportional to the product $\alpha \beta$, see Equation 5) that are obtained by solution of the scaled form of Equations (2) and (3) subject to the initial conditions given above are shown in Figure 2 for various values of $\lambda$. It is worthy of note that in the simplified model that we have developed, the only parameter that determines the behavior of the reactor is $\lambda$. This parameter captures the effects of melt size relative to its surface area, reaction rate constant, pyrolysis rate constant, and the concentrations of methane and metal oxide on the rate at which chemical conversion takes place in the reactor.

Figure 2 shows that the metal oxide concentration in the melt decreases monotonically with time, and the amount of carbon deposited on the melt increases with time. The rate of production of carbon monoxide produced is initially zero and increases with time as the reaction between the deposited carbon and the metal oxide progresses. As the metal oxide is depleted, the reaction finally slows down and stops. Figure 2 shows that the rate of production of CO attains a maximum before it eventually decreases to zero. The exact shape of the curves, especially that for the the rate of production of $\mathrm{CO}$, is sensitive to the value of $\lambda$. $\lambda$ is a measure of the relative importance of the rate of production of carbon by the surface pyrolysis of methane to its rate of consumption by reaction with the metal oxide. The former rate is constant for typical operating conditions of fixed temperature, pressure, and sufficient flow rate to maintain a 
constant methane concentration. The latter rate varies depending on the accumulation or depletion of carbon and the availability of the metal oxide in the melt. So, the magnitude of $\lambda$ indicates the relative dominance of the two rates in a competitive process; i.e., small lambda means relatively small carbon deposition rates, and vice versa.
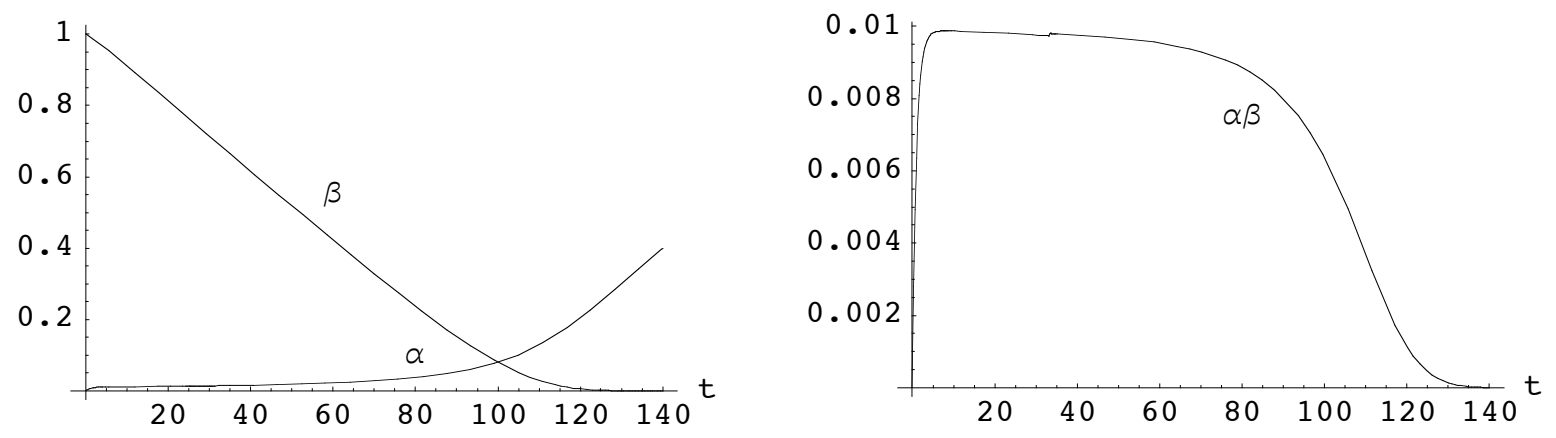

(a) $\lambda=0.01$
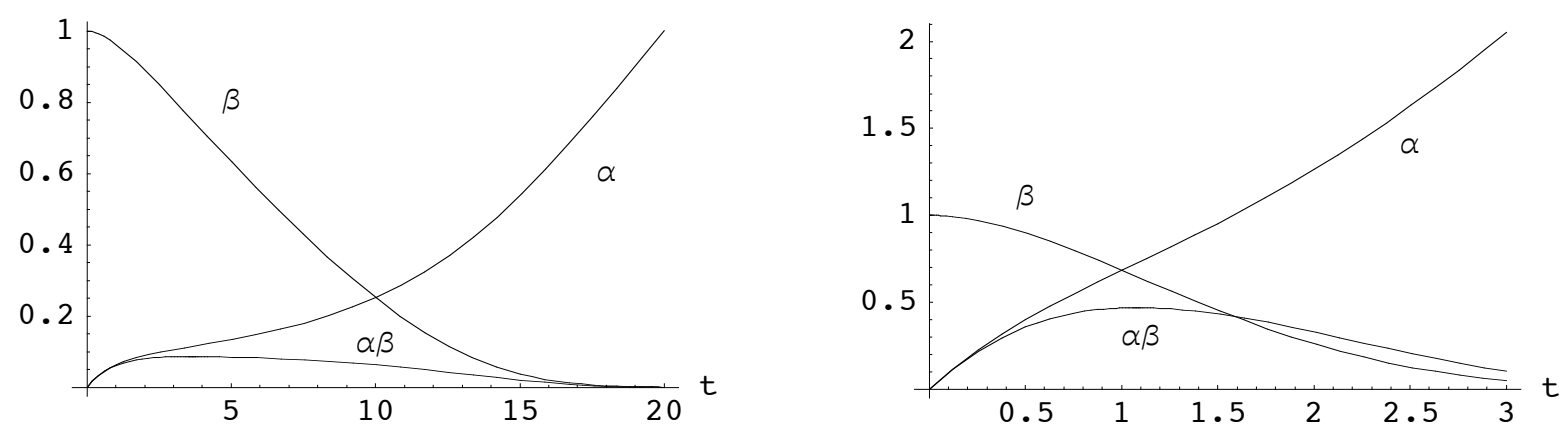

(b) $\lambda=0.1$

(c) $\lambda=1$

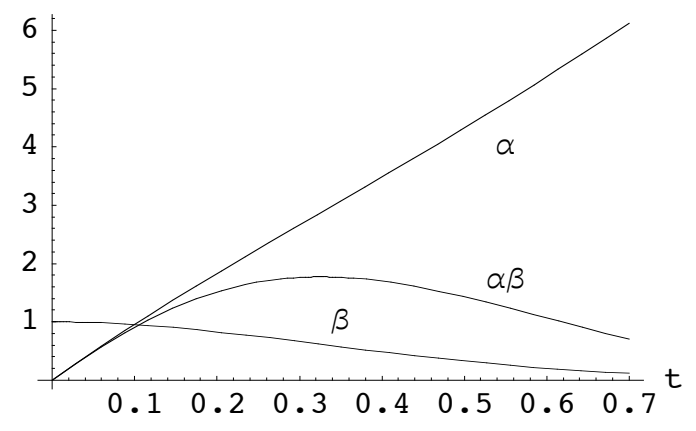

(d) $\lambda=10$

FIGURE 2: Plot of the scaled concentrations of carbon $(\alpha)$ and metal oxide $(\beta)$ in the melt and the scaled rate of production of carbon monoxide $(\alpha \beta)$ versus scaled time $(t)$ for various values of $\lambda$.

Since the amount of carbon in the melt increases with time, there is unreacted carbon that is left behind in the melt after the reaction is completed. As the spent regolith is dumped out of the reactor, the unreacted carbon is a wastage of precious resources since carbon is a scarce quantity on the moon. A useful strategy to minimize the amount of unreacted carbon is to turn the supply of methane off at an appropriate time before the reaction is completed, such that the residual amount of carbon present in the melt at that time is sufficient to react with the remaining amount of the metal oxide. After methane is turned off, the product gases must be recovered by suitable means, such as by using sweep gases. 


\section{CONCLUSION}

We have developed a model which describes the chemical conversion of lunar regolith in a carbothermal reactor to produce carbon monoxide, and eventually oxygen. Methane flows over a molten regolith zone in the reactor continuously at a fixed temperature and pressure to maintain its concentration. Our model assumes a two-step process for the chemical conversion. First, methane is pyrolyzed as it comes in contact with the hot, molten regolith surface. The solid carbon formed after pyrolysis is deposited on the melt, and mixes with it uniformly. The second step is the reaction which takes place between carbon and the metal oxide in the melt to yield carbon monoxide that bubbles out of the melt. Our model shows that a single parameter captures the effects of melt size relative to its surface area, reaction rate constant, pyrolysis rate constant, and the concentrations of methane and metal oxide on the rate at which chemical conversion takes place in the reactor. Validation of the model predictions with experimental results are being performed.

\section{NOMENCLATURE}

$\begin{array}{ll}\mathrm{a} & =\text { stoichiometric coefficient in chemical reaction in } \mathrm{Eq}(1) \\ \mathrm{A} & =\text { melt surface area } \\ \mathrm{C}_{m} & =\text { concentration of methane }\left(\text { mole } / \mathrm{m}^{3}\right) \\ \mathrm{k} & =\text { reaction rate constant }\left(\mathrm{m}^{3} /(\text { mole } \mathrm{s})\right) \\ \mathrm{r}_{p} & =\text { flux of carbon from pyrolysis }\left(\text { mole } /\left(\mathrm{m}^{2} \mathrm{~s}\right)\right) \\ \mathrm{t}^{*} & =\text { time }(\mathrm{s}) \\ \mathrm{V} & =\text { melt volume }\left(\mathrm{m}^{3}\right) \\ \mathrm{X}_{C O} & =\text { number of moles of carbon monoxide }(\text { mole }) \\ \alpha, \alpha^{*} & =\text { concentration of carbon in the melt, scaled }(\alpha) \text { and physical }\left(\alpha^{*}, \mathrm{~mole} / \mathrm{m}^{3}\right) \\ \beta, \beta^{*} & =\text { concentration of metal oxide in the melt, scaled }(\beta) \text { and physical }\left(\beta^{*}, \mathrm{~mole}^{3}\right) \\ \beta_{0} & =\text { initial concentration of metal oxide in the melt }\left(\text { mole } / \mathrm{m}^{3}\right) \\ \gamma & =\text { proportionality constant in the expression for rate of pyrolysis of methane }\end{array}$

\section{ACKNOWLEDGMENTS}

R. Balasubramaniam and U. Hegde were supported by a Cooperative Agreement NCC3-975 from NASA Glenn Research Center to the National Center for Space Exploration Research. The authors would like to thank K. Sacksteder and D. Linne of NASA Glenn Research Center and E. Santiago-Maldonaldo of NASA Kennedy Space Center for discussions and comments during the course of the investigation.

\section{REFERENCES}

ORBITEC, “Carbothermal Reduction of Lunar Regolith,” Phase 1 Final Report OTC-GS0167-FR-06-1, Orbital Technologies Corporation (ORBITEC), Madison, Wisconsin, USA, 29 September 2006.

Rosenberg, S.D., Beegle, R.L., Jr, Guter, G.A., Miller, F.E., Rothenberg, M., "The onsite manufacture of propellant oxygen from lunar resources," In NASA. Johnson Space Center, Space Resources, Volume 3: Materials, 162-185, 1991 (SEE N93-16875 05-91).

Sanders, G.B., Larson, W.E., and Sacksteder, K.R., "NASA Overview of In-Situ Resource Utilization (ISRU) Architecture." National Academy of Sciences Workshop on Research Enabled by the Lunar Environment, June 14, 2007, http://www7.nationalacademies.org/ssb/1LunarWorkshop_Sanders.pdf, accessed September 19, 2007.

Taylor, L.A. and Carrier, W.D. III, "Production of Oxygen on the Moon: Which Processes are Best and Why," AIAA Journal, 30(12), 2858-2863 (1992).

Tripuraneni-Kilby, K.C., Centeno, L., Doughty, G., Mucklejohn, S., and Fray, D.J., "The Electrochemical Production of Oxygen and Metal via the FFC-Cambridge Process," http://www.lpi.usra.edu/meetings/roundtable 2006/pdf/tripuraneni.pdf, accessed September 17, 2007.

Wang, Y. and Yuan, Z., "Reductive kinetics of the reaction between a natural ilmenite and carbon," Int. J. Miner. Process. 81, 133-140 (2006). 